\title{
KRAS Protein Variant
}

National Cancer Institute

\section{Source}

National Cancer Institute. KRAS Protein Variant. NCI Thesaurus. Code C98362.

A variation in the amino acid sequence for the GT Pase KRas protein. 